\title{
Ginsenoside composition of Panax ginseng flower extracts obtained using different high hydrostatic pressure extraction conditions
}

\author{
Hyun Soo Kim • Gyu Ri Kim • Donghyun Kim • Cheng-Yi Zhang • Eun-Soo Lee • Nok Hyun Park • Junseong Park • \\ Chang Seok Lee $\cdot$ Moon Sam Shin
}

Received: 1 February 2019 / Revised: 26 February 2019 / Accepted: 26 February 2019

(c) Korean Society for Plant Biotechnology

\begin{abstract}
Ginsenosides are active constituents of ginseng (Panax ginseng) that have possible anti-aging, physiological and pharmacological activities, such as anti-cancer and anti-inflammatory effects. Although the ginseng root is generally used more often than the aerial parts for medicinal purposes, the flowers also contain numerous ginsenosides, including $\mathrm{Rb} 2, \mathrm{Rc}, \mathrm{Rd}$, Re and $\mathrm{Rg} 1$. Therefore, an extract from the flowers of the $P$. ginseng could have the pharmacological efficacy of bioactive compounds including ginsenosides. The high hydrostatic pressure extraction (HHPE) is a method that is used for the efficient extraction of bioactive compounds from plant materials. In this study, we compared the yield of ginsenosides from ginseng flowers under different conditions of extraction pressure and time of HHPE. The results indicate that the total yield of the ginsenosides improved as the pressure increased from 0.1 to $80 \mathrm{MPa}$ and treatment duration increased to 24 hours. In addition, the ginsenoside extracts from HHPE at $80 \mathrm{MPa}$, which possessed a higher total ginsenoside concentration, decreased the viability of the primary human epidermal keratinocytes (HEKs) significantly than the ginsenoside extracts from HHPE at $0.1 \mathrm{MPa}$. Collectively, we found that the method of HHPE that was performed for 24 hours at 80 $\mathrm{MPa}$ showed the highest yield of ginsenosides from the flowers of $P$. ginseng. In addition, our study provides a
\end{abstract}

${ }^{\dagger}$ These authors contributed equally to this work.

H. S. Kim ${ }^{\dagger} \cdot$ D. Kim $\cdot$ C.-Y. Zhang $\cdot$ E.-S. Lee $\cdot$ N. H. Park Amorepacific R\&D Center, Yongin 17074, Korea

G. R. $\mathrm{Kim}^{\dagger} \cdot$ C. S. Lee $\cdot$ M. S. Shin $(\varangle)$

Department of Beauty and Cosmetic Science, Eulji University, Seongnam 13135, Korea

e-mail: msshin@eulji.ac.kr

J. Park

Department of Engineering Chemistry, Chungbuk National

University, Cheongju 54896, Korea foundation for the efficient extraction of ginsenosides, which had a potent bioactivity, from flowers of $P$. ginseng through HHPE.

Keywords Ginsenoside, Panax ginseng, High hydrostatic Pressure extraction (HHPE), Cell viability

\section{Introduction}

Pharmacological studies of active ingredients in plants of the genus Panax (ginseng) have identified more than 30 kinds of dammarane-type triterpene oligoglycosides, more generally known as ginsenosides. The ginsenosides $\mathrm{Rb}$, $\mathrm{Rb} 2, \mathrm{Rc}, \mathrm{Rd}, \mathrm{Re}$, and $\mathrm{Rg} 1$ are the principal constituents, and there are also polysaccharides, phenolics, lignins, polyacetylenes, and acidic peptides (Park JD 1996; Jia et al. 2009; Kazuhiro et al. 1991; Ali et al. 2006). Various studies have been conducted to understand the pharmacological mechanisms of ginseng and ginsenosides in cardiovascular disease, diabetes mellitus, cancers and stress. Recently, the anti-inflammatory role of ginseng and ginsenosides in inflammatory responses are introduced (Kim et al. 2017). Especially, ginsenoside Re showed the skin protective effect, resulting from the inhibition of UVB-induced oxidative responses (Shin et al. 2018).

Most ginseng studies have examined extracts from the roots, but extracts from the aerial parts also contain ginsenosides, although their exact ginsenoside compositions are somewhat different (Tung et al. 2010; Tung et al. 2012; Yahara et al. 1979). Therefore, an extract of aerial parts, such as flowers, of $P$. ginseng is a promising source for bioactive compounds.

Extraction is the first step for the isolation and purification of bioactive compounds from natural products (Lee et al. 2011). Traditional extraction methods generally use heat or 
stirring to improve the solubility and isolation of the desired compounds, but these procedures are often time consuming and have low efficiency (Chen et al. 2009). The high hydrostatic pressure extraction (HHPE) method, also known as cold isostatic pressure treatment, is widely used in the food industry because it can extend shelf-life by reducing the numbers of bacteria and the activity of enzymes (Lee et al. 2011). This method can be also used to extract compounds from various plant or herbal materials at room temperature (Shouqin et al. 2005).

In the present study, we compared the composition of ginsenosides isolated from flowers of $P$. ginseng by variation of HHPE conditions (pressure or time). In addition, we determined in vitro viability effects of extracts isolated under different HHPE conditions to epidermal keratinocytes. This study is the first to examine the extraction of ginsenosides, which has a biological efficacy, from flowers of $P$. ginseng using HHPE.

\section{Materials and Methods}

\section{Materials}

Six-year-old flowers of cultivated $P$. ginseng from Sangju, Korea were dried in an oven at $45^{\circ} \mathrm{C}$ until a constant weight was obtained, and then powdered in a mill and passed through a 40-mesh sieve. Ginsenoside standards (Rb1, Rb2, $\mathrm{Rc}, \mathrm{Rd}, \mathrm{Re}$, and Rg1) were from Embo Laboratory (Daejeon, Korea), high performance liquid chromatography (HPLC) -grade acetonitrile was from Fisher Scientific Company (Pittsburgh, PA, USA), and distilled water was from Millipore Direct-Q (Millipore, Mississauga, Canada).

Analysis of ginsenosides by high-performance liquid chromatography

Ginsenoside analysis was performed using HPLC (Alliance 2695 system, Waters Co., Milford, MA, USA) with a photodiode array (PDA) detector (Waters 2998). Empower Pro 3 software (Build 3471) was used for gradient programming and integration of absorption peaks. Separation was performed on a C18 reversed-phase column (Mightisil RP-18 GP, $250 \times$ $4.6 \mathrm{~mm}$; Kanto Chemical, Japan) at a column temperature of $30^{\circ} \mathrm{C}$. The gradient consisted of water (A) and acetonitrile (B) as solvents. To determine ginsenosides $\mathrm{Rb} 1, \mathrm{Rb} 2$, $\mathrm{Rc}$, and Rd, the regimen was 0-10 min, $65-55 \% \mathrm{~A} ; 10-23 \mathrm{~min}$, 55-0\% A; 23-25 min, 0-65\% A; to determine ginsenosides Re and Rg1, the regimen was $0-55 \mathrm{~min}, 80-80 \%$ A; $55-65$ $\min , 80-20 \%$ A; $65-75 \min , 20-20 \%$ A; 75-80 min, 20-80\% A. The injection volume was $10 \mu \mathrm{L}$ and the flow rate was $1 \mathrm{~mL} / \mathrm{min}$. An extract of $10 \mathrm{mg}$ was weighed and dissolved in $1 \mathrm{~mL}$ of HPLC grade methanol for quantitation of the different ginsenosides.

\section{HHPE and sample preparation}

HHPE was performed in a UHP machine TFS-2L (Toyo Koatsu Co. Ltd, Hiroshima, Japan), which allowed control of pressure, temperature, and time. A total of $100 \mathrm{~g}$ of dried flowers were added to a vacuum bag containing $70 \%(\mathrm{~V} / \mathrm{V})$ ethanol/water in a solid/liquid ratio of $1: 10$, and controlled extractions were carried out at $0.1 \mathrm{MPa}, 10 \mathrm{MPa}, 20 \mathrm{MPa}$, $40 \mathrm{MPa}$, and $80 \mathrm{MPa}$, with a fixed time of $24 \mathrm{~h}$ and a fixed temperature of $30^{\circ} \mathrm{C}$. After cooling to room temperature, the extraction solution was vacuum filtered, evaporated under reduced pressure at $45^{\circ} \mathrm{C}$, and the residue was then dissolved in methanol. The sample solution was passed through a $0.45 \mu \mathrm{m}$ filter prior to analysis. The ginsenoside standards (Rb1, Rb2, Rc, Rd, Re, Rg1) were prepared in HPLC-grade methanol at different concentrations for creation of standard curves. Namely, concentrations of ginsenosides were determined by standard curves prepared by injecting different concentrations of ginsenoside standards (Popovich et al. 2004).

\section{Cell culture}

Primary human epithelial keratinocytes (HEKs) were grown in KBM-Gold medium (Lonza \#0019251) containing antibiotics (1\% Penicillin/Streptomycin) and supplements at $37^{\circ} \mathrm{C}$ under $5 \% \mathrm{CO}_{2}$. Before cells were seeded, the bottom of the culture flask was coated with a solution of type IV collagen solution $(0.1 \mathrm{mg} / \mathrm{mL})$. Keratinocytes were serially passaged at $70 \sim 80 \%$ confluence, and experiments were carried out using subconfluent cells at passage number 3 to 5 , at which time they were proliferating actively.

\section{Cell viability analysis}

HEKs were cultivated on 96 well plates $\left(1 \times 10^{4}\right.$ cells/well $)$ for $24 \mathrm{~h}$. Samples of the extracts were added to cells at concentrations of $3,6,12,25,50 \mu \mathrm{g} / \mathrm{mL}$, with 3 replicates per concentration. After $24 \mathrm{~h}$, the media was removed and $100 \mathrm{ul}$ of $10 \%$ CCK-8 solution (diluted in the culture media) was added to each well. After incubation for $2 \mathrm{~h}$ at $37^{\circ} \mathrm{C}$, absorbance at $450 \mathrm{~nm}$ was measured using a microplate reader. Cell viability (CV) rate was calculated as the following; 


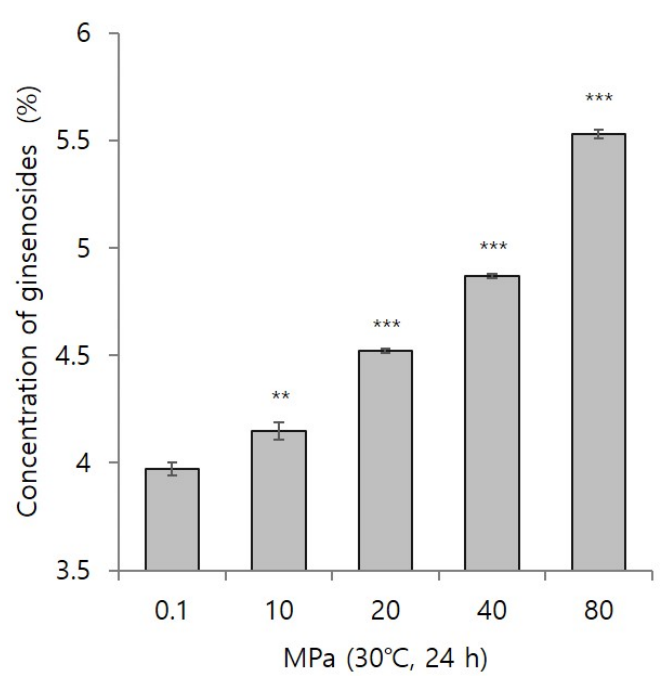

Fig. 1 Effect of the HHPE pressure on extraction of total ginsenosides at $30^{\circ} \mathrm{C}$ for 24 hours. ${ }^{*} p<0.05$, ** $p<0.01$, *** $p<0.001$. vs control group (0.1 MPa)

$\mathrm{CV}(\%)=\left\{\left(\mathrm{A}_{\text {sample }}-\mathrm{A}_{\mathrm{b}}\right) /\left(\mathrm{A}_{\mathrm{c}}-\mathrm{A}_{\mathrm{b}}\right)\right\} \times 100\left(\mathrm{~A}_{\text {sample }}\right.$; the absorbance of extracts-treated group., $A_{b}$; the absorbance of blank group., $\mathrm{A}_{c}$; the absorbance of control group. Cell viability was determined using a cell cytotoxicity kit (CCK-8), as described by the manufacturer (DOJINDO, Tokyo, Japan).

\section{Statistical analysis}

Data are expressed as means \pm standard deviations (SDs), and Student's $t$-test was used for statistical comparisons. A $p$-value below 0.05 was considered statistically significant.

\section{Results and Discussion}

Extraction pressure is one of the most important features affecting the efficiency of HHPE. We initially analyzed the yield of ginsenosides from flowers of $P$. ginseng, as

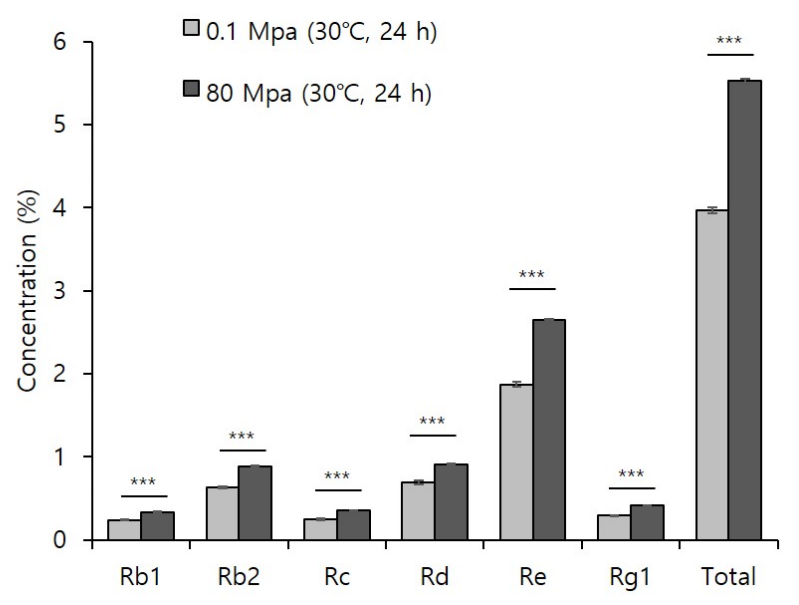

Fig. 2 Effect of HHPE pressure on extraction of 6 specific ginsenosides (Rb1, Rb2, Rc, Rd, Re, and $\mathrm{Rg} 1)$ at $30^{\circ} \mathrm{C}$ for 24 hours. * $p<0.05, * * p<0.01$, *** $p<0.001$

described in material and method section. In Figure 1 and Table 1, the total concentration of ginsenosides was about 1.392-fold higher for a pressure of $80 \mathrm{MP}$ than $0.1 \mathrm{MP}$. The extraction yields of all ginsenosides ( $\mathrm{Rb} 1, \mathrm{Rb} 2, \mathrm{Rc}$, $\mathrm{Rd}, \mathrm{Re}$, and $\mathrm{Rg} 1$ ) increased as pressure increased (Fig. 2). In particular, the content of ginsenoside Re, the most abundant form (accounting for $47.92 \%$ of all ginsenosides) increased by 1.41 -fold as the pressure increased from 0.1 to $80 \mathrm{MPa}$.

We also examined the effect of extraction time on the efficiency of HHPE. Thus, we performed extractions for 1 , $6,12,24$ and $48 \mathrm{~h}$ at a pressure of $80 \mathrm{MPa}$ and a temperature of $30^{\circ} \mathrm{C}$ (Fig. 3 and Table 2). The results show that the total yield increased as the extraction time increased, although the concentration of total ginsenosides was slightly lower after $48 \mathrm{~h}$ than $24 \mathrm{~h}$. Therefore, a 24 $\mathrm{h}$ HHPE extraction time provides the best extraction of total ginsenosides from ginseng flowers.

Previous reports showed that bioactive ginsenosides,

Table 1 Concentrations (\%) of 6 ginsenosides extracted through HHPE under different pressures at $30^{\circ} \mathrm{C}$ for 24 hours

\begin{tabular}{lccccc}
\hline Ginsenosides & \multicolumn{5}{c}{ Pressure } \\
\cline { 2 - 6 }$(\%)$ & $0.1 \mathrm{MPa}$ & $10 \mathrm{MPa}$ & $20 \mathrm{MPa}$ & $40 \mathrm{MPa}$ & $80 \mathrm{MPa}$ \\
\hline $\mathrm{Rb} 1$ & $0.24 \pm 0.01$ & $0.25 \pm 0.01$ & $0.29 \pm 0.01$ & $0.30 \pm 0.01$ & $0.33 \pm 0.01$ \\
$\mathrm{Rb} 2$ & $0.63 \pm 0.01$ & $0.74 \pm 0.01$ & $0.74 \pm 0.01$ & $0.87 \pm 0.01$ & $0.88 \pm 0.01$ \\
$\mathrm{Rc}$ & $0.25 \pm 0.01$ & $0.26 \pm 0.01$ & $0.31 \pm 0.01$ & $0.32 \pm 0.01$ & $0.35 \pm 0.01$ \\
$\mathrm{Rd}$ & $0.69 \pm 0.02$ & $0.65 \pm 0.02$ & $0.78 \pm 0.01$ & $0.83 \pm 0.01$ & $0.91 \pm 0.01$ \\
$\mathrm{Re}$ & $1.87 \pm 0.01$ & $1.93 \pm 0.03$ & $2.08 \pm 0.01$ & $2.19 \pm 0.01$ & $2.65 \pm 0.01$ \\
$\mathrm{Rg} 1$ & $0.29 \pm 0.01$ & $0.33 \pm 0.01$ & $0.32 \pm 0.01$ & $0.35 \pm 0.01$ & $0.41 \pm 0.01$ \\
Total & $3.97 \pm 0.03$ & $4.15 \pm 0.04$ & $4.52 \pm 0.01$ & $4.87 \pm 0.01$ & $5.53 \pm 0.02$ \\
\hline
\end{tabular}


Table 2 Concentrations (\%) of 6 ginsenosides extracted through HHPE for different times at $30^{\circ} \mathrm{C}$ and $80 \mathrm{MPa}$

\begin{tabular}{lccccc}
\hline Ginsenosides & \multicolumn{5}{c}{ times } \\
\cline { 2 - 6 }$(\%)$ & $1 \mathrm{~h}$ & $6 \mathrm{~h}$ & $12 \mathrm{~h}$ & $24 \mathrm{~h}$ & $48 \mathrm{~h}$ \\
\hline $\mathrm{Rb} 1$ & $0.19 \pm 0.01$ & $0.27 \pm 0.01$ & $0.29 \pm 0.01$ & $0.33 \pm 0.01$ & $0.35 \pm 0.01$ \\
$\mathrm{Rb} 2$ & $0.61 \pm 0.02$ & $0.69 \pm 0.01$ & $0.72 \pm 0.01$ & $0.88 \pm 0.01$ & $0.86 \pm 0.01$ \\
$\mathrm{Rc}$ & $0.21 \pm 0.01$ & $0.28 \pm 0.01$ & $0.30 \pm 0.01$ & $0.35 \pm 0.01$ & $0.35 \pm 0.01$ \\
$\mathrm{Rd}$ & $0.58 \pm 0.01$ & $0.77 \pm 0.01$ & $0.81 \pm 0.01$ & $0.91 \pm 0.01$ & $0.89 \pm 0.01$ \\
$\mathrm{Re}$ & $1.97 \pm 0.03$ & $2.21 \pm 0.01$ & $2.20 \pm 0.02$ & $2.65 \pm 0.01$ & $2.49 \pm 0.01$ \\
$\mathrm{Rg} 1$ & $0.34 \pm 0.01$ & $0.34 \pm 0.01$ & $0.37 \pm 0.01$ & $0.41 \pm 0.01$ & $0.40 \pm 0.02$ \\
Total & $3.90 \pm 0.01$ & $4.56 \pm 0.02$ & $4.69 \pm 0.02$ & $5.53 \pm 0.02$ & $5.34 \pm 0.02$ \\
\hline
\end{tabular}

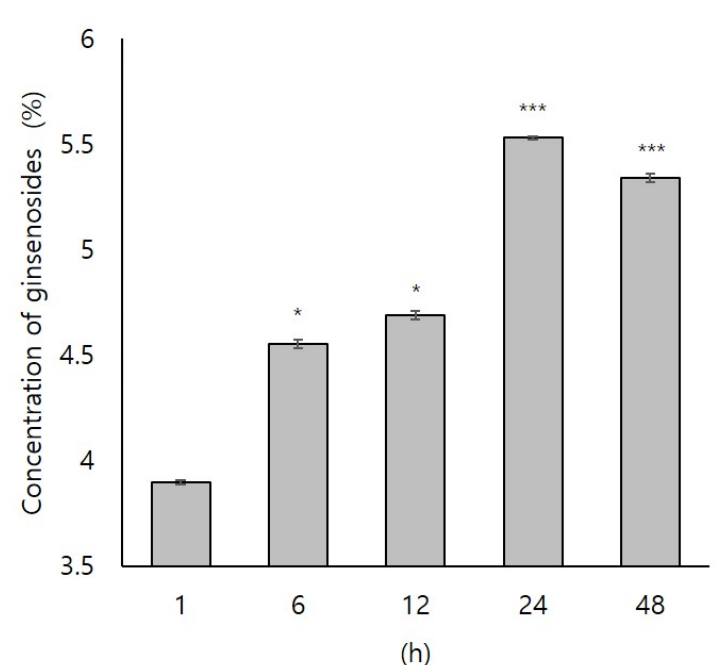

Fig. 3 Effect of HHPE duration on extraction of ginsenosides at $30^{\circ} \mathrm{C}$ and $80 \mathrm{MPa} . * p<0.05, * * p<0.01, * * * p<0.001$. $v s$ control group $(1 \mathrm{~h})$

such as Rc and Re, affect the viability, physiological function, and general condition of epidermal keratinocytes (Oh et al. 2016; Oh et al. 2017; Lim et al. 2016). Typically, the excessive concentration of almost all ginsenosides could increase the cytotoxicity, although the specific concentration of several ginsenosides have a cell proliferative efficacy (Chen et al. 2016; Lee et al. 2012). Thus, to confirm the relative concentration of total ginsenosides though the cell viability, we investigated the effects of total ginsenosides isolated from flower extracts using two HHPE protocols (0.1 $\mathrm{MPa}, 30^{\circ} \mathrm{C}, 24 \mathrm{~h}[\mathrm{GF} 1]$ and $80 \mathrm{MPa}, 30^{\circ} \mathrm{C}, 24 \mathrm{~h}$ [GF2]) at five concentrations $(3,6,12,25$ and $50 \mu \mathrm{g} / \mathrm{mL})$ on the viability of primary human epidermal keratinocytes (HEKs). Expectedly, the results indicate the GF2 extract, which had a higher total ginsenoside concentration, decreased cell viability significantly more than the GF1 extract (Fig. 4). Therefore, we suggested that the total ginsenosides isolated from flower extracts using GF2 HHPE protocol are bioactive because the higher level of total ginsenosides in

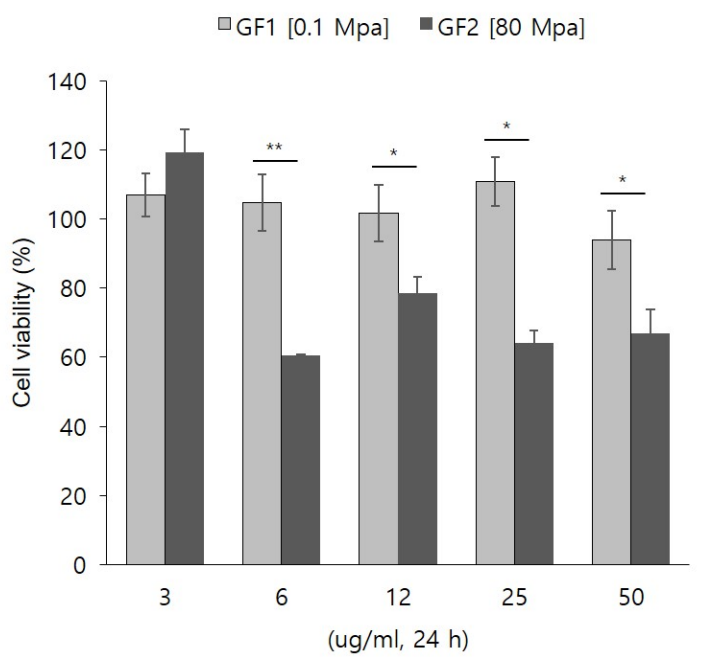

Fig. 4 Effect of different levels of ginsenosides extracted using 2 regimens (GF1: $0.1 \mathrm{MPa}, 30^{\circ} \mathrm{C}, 24$ hours; GF2: $80 \mathrm{MPa}$, $30^{\circ} \mathrm{C}, 24$ hours) on viability of HEKs. Values are means $\pm \mathrm{SD}$ of three independent experiments. ${ }^{*} p<0.05,{ }^{* *} p<0.01$, *** $p<0.001$

GF2 have more potent effect on cell viability. However, further experiments are needed to confirm whether the other factors except for ginsenosides affects on the cell viability.

In the current study, we examined the effect of different HHPE variables (pressure and time) on the yield of ginsenosides from ginseng flowers. HHPE conducted for $24 \mathrm{~h}$ at $80 \mathrm{MPa}$ provided the highest yield of ginsenosides. However, all extractions were performed at $30^{\circ} \mathrm{C}$, and we have no data regarding the impact of temperature on ginsenoside extraction. In addition, our results indicated that extracts from HHPE at $80 \mathrm{MPa}$ had a greater impact on the viability of HEKs than those from HHPE at $0.1 \mathrm{MPa}$. These results suggest that ginsenosides (especially Re) extracted from the flowers of $P$. ginseng under specific HHPE conditions may influence skin physiology, by strengthening the skin barrier or promoting epidermal differentiation. In 
agreement, our preliminary data indicated that ginseng flower extracts isolated using HHPE increased the expression of genes related to the protective efficacy of the skin barrier (data not shown). In conclusion, ginsenosides extracted from ginseng flowers, using an HHPE protocol at $80 \mathrm{MPa}$ for $24 \mathrm{~h}$, have potential use as bioactive compounds.

\section{Acknowledgement}

This study was supported by Amorepacific corporation and by the Bio \& Medical Technology Development Program of the National Research Foundation(NRF) funded of the Ministry of Science \& ICT (2017M3A9D8048416).

\section{References}

Ali MB, Singh N, Shohael AM, Hahn EJ, Paek K-Y (2006). Phenolics metabolism and lignin synthesis in root suspension cultures of Panax ginseng in response to copper stress. Plant Sci 171:147-154

Chen R, Meng F, Zhang S, Liu Z (2009). Effects of ultrahigh pressure extraction conditions on yields and antioxidant activity of ginsenoside from ginseng. Sep Purif Technol 66:340-346

Chen Y, Liu ZH, Xia J, Li XP, Li KQ, Xiong W, Li J, Chen DL (2016). 20(S)-ginsenoside Rh2 inhibits the proliferation and induces the apoptosis of KG-1a cells through the Wnt/ $\beta$ -catenin signaling pathway. Oncol Rep 36:137-46

Jia L, Zhao Y, Liang XJ (2009). Current evaluation of the millennium phytomedicine-ginseng (II): Collected chemical entities, modern pharmacology, and clinical applications emanated from traditional Chinese medicine. Curr Med Chem 16:2924-2942

Kazuhiro H, Makoto M, Kaoru N, Yukinobu I, Hiroshi M (1991). Polyacetylenes from the roots of Panax ginseng. Phytochemistry 30:3327-3333

Kim JH, Yi YS, Kim MY, Cho JY (2017). Role of ginsenosides, the main active components of Panax ginseng, in inflammatory responses and diseases. J Ginseng Res 41:435-443

Lee HS, Lee HJ, Yu HJ, Ju do W, Kim Y, Kim CT, Kim CJ, Cho YJ, Kim N, Choi SY, Suh HJ (2011). A comparison between high hydrostatic pressure extraction and heat extraction of ginsenosides from ginseng (Panax ginseng CA Meyer). J Sci Food Agric 91:1466-1473

Lee KW, Jung SY, Choi SM, Yang EJ (2012). Effects of ginsenoside Re on LPS-induced inflammatory mediators in BV2 microglial cells. BMC Complement Altern Med 12:196

Lim HW, Kim K, Lim CJ (2016). Contribution of ginsenoside Re to cellular redox homeostasis via upregulating glutathione and superoxide dismutase in $\mathrm{HaCaT}$ keratinocytes under normal conditions. Pharmazie 71:413-419

Oh Y, Lim HW, Kim K, Lim CJ (2016). Ginsenoside Re improves skin barrier function in HaCaT keratinocytes under normal growth conditions. Biosci Biotechnol Biochem 13:1-3

Oh Y, Lim HW, Park KH, Huang YH, Yoon JY, Kim K, Lim CJ 2017). Ginsenoside Rc protects against UVBinduced photooxidative damage in epidermal keratinocytes. Mol Med Rep 16:2907-2914

Park JD (1996). Recent studies on the chemical constituents of Korean ginseng (Panax ginseng CA Meyer). Korean J Ginseng Sci 20:389-415

Popovich DG, Kitts DD (2004). Generation of ginsenosides Rg3 and Rh2 from North American ginseng. Phytochemistry 65:337-44

Shin D, Moon HW, Oh Y, Kim K, Kim DD, Lim CJ (2018). Defensive Properties of Ginsenoside Re against UV-B-Induced Oxidative Stress through Up-Regulating Glutathione and Superoxide Dismutase in HaCaT Keratinocytes. Iran J Pharm Res 17:249-260

Shouqin Z, Jun X, Changzheng W (2005). High hydrostatic pressure extraction of flavonoids from propolis. $\mathrm{J}$ Chem Technol Biotechnol 80:50-54

Tung NH, Song, GY, Nhiem NX, Ding Y, Tai BH, Jin LG, Lim, CM, Hyun, JW, Park CJ, Kang HK, Kim YH (2010). Dammarane-type saponins from the flower buds of Panax ginseng and their intracellular radical scavenging capacity. J Agric Food Chem 58:868-874

Tung NH, Song GY, Woo S, Hyun JW, Koh YS, Kang HK, Shoyama Y, Kim YH (2012). Ginsenosides from the leaves and flower buds of panax ginseng and their pharmacological effects. Curr Bioact Comp 8:159-166

Yahara S., Kaji K., Tanaka O (1979). Further Study on Darnmarane-Type Saponins of Roots, Leaves Flower-Buds, and Fruits of Panax ginseng CA Meyer. Chem Pharm Bull 27:88-92 